\title{
Randomised controlled trial for emphysema with a selective agonist of the $\gamma$-type retinoic acid receptor
}

\author{
Jan Stolk, Robert A. Stockley, Berend C. Stoel, Brendan G. Cooper, \\ Eeva Piitulainen, Niels Seersholm, Kenneth R. Chapman, Jonathan G.W. Burdon, \\ Marc Decramer, Raja T. Abboud, Gregorius P.M. Mannes, Emiel F. Wouters, \\ Jeffrey E. Garrett, Juan C. Barros-Tizon, Erich W. Russi, David A. Lomas, \\ William A. MacNee and Alexis Rames
}

ABSTRACT: Palovarotene is an oral $\gamma$-selective retinoid agonist. In animal emphysema models, palovarotene reduced inflammation, promoted structural repair and functional improvement.

REPAIR (Retinoid treatment of Emphysema in Patients on the $\alpha_{1}$-antitrypsin International Registry), was an investigator-initiated, double-blind, placebo-controlled randomised study to assess the safety and efficacy of $5 \mathrm{mg} \cdot \mathrm{day}^{-1}$ palovarotene given for 1 year to 262 patients with severe $\alpha_{1}$-antitrypsin deficiency and emphysema confirmed by computed tomography. Change in volume-adjusted 15th percentile point lung density from baseline in 1 year was the primary endpoint; functional end-points were also regularly assessed.

We randomly assigned 133 and 129 patients to placebo or palovarotene, respectively. Both groups were well matched for all baseline characteristics, including respiratory medications. $88 \%$ and $85 \%$ of patients completed 1 year of treatment with placebo and palovarotene, respectively. Palovarotene was generally well tolerated. In the study completers population, the placebocorrected difference of lung density was $-0.45 \mathrm{HU}$ at week $28(p=0.64)$ and $-0.25 \mathrm{HU}$ at week 52 $(p=0.94)$. A nonsignificant treatment difference in most functional parameters of the lung in favour of the drug was observed over time suggesting potential pharmacological effects of palovarotene.

Palovarotene $5 \mathrm{mg} \cdot \mathrm{day}^{-1}$ over $1 \mathrm{yr}$ failed to show a significant benefit on lung density in moderate-to-severe emphysema secondary to severe $\alpha_{1}$-antitrypsin deficiency.

KEYWORDS: $\alpha_{1}$-antitrypsin, chronic obstructive pulmonary disease treatment, clinical trials, densitometry, emphysema, retinoids

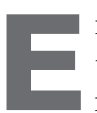

mphysema most commonly results from tobacco-induced inflammation and/or chronic proteinase-antiprotease imbalance in the lung $[1,2]$. The resultant loss of lung parenchyma reduces the surface area available for gas exchange, causing impaired oxygen transfer with increased pulmonary vascular resistance and right heart dysfunction. The loss of lung matrix reduces lung elastic recoil pressure and results in mechanical failure of the lung. Ultimately, these changes lead to hyperinflation of the lung, airflow limitation, and ventilation/perfusion mismatch. These progressive changes have been considered irreversible and therapies have focused on smoking cessation, symptomatic relief and reduction of complications [3]. $\alpha_{1}$-antitrypsin of ZZ genotype (AATD) is a genetic disorder that results in severe deficiency of $\alpha_{1}$-antitrypsin (AAT) in blood and tissue fluids. AAT is a protease inhibitor of broad specificity and neutrophil elastase is its main target. In smokers with AATD the disorder is clinically characterised by early-onset emphysema classically present in the lower lung lobes and considered more rapidly progressive than that seen in non-AATD chronic obstructive pulmonary disease (COPD)/emphysema patients who smoke. Currently, there are no treatments that restore lung function in emphysema. Intravenous augmentation therapy is used in patients with AATD to reduce the rate of loss of lung tissue, and this therapy has been suggested to be
AFFILIATIONS

For author affiliations, see the Acknowledgements section.

CORRESPONDENCE J. Stolk

Dept of Pulmonology Leiden University Medical Center Leiden

The Netherlands

E-mail: j.stolk.long@lumc.nl

Received:

Sept 172011

Accepted after revision: Dec 062011

First published online:

Jan 262012 
efficacious based on a combination of data from two randomised, placebo-controlled trials [4].

All-trans-retinoic acid (ATRA) and other vitamin A derivatives or retinoids are ligands for two classes of nuclear hormone receptors, RAR and RXR. These receptors, when bound to retinoic acid or other retinoids, activate and/or repress gene expression to modulate structure and function of tissues. Temporal and spatial regulation of RAR receptor expression is required for proper tissue development and tissue maintenance. For example, RAR- $\gamma$ is highly expressed during embryonic lung development and promotes alveolar septation. In addition, retinoids increase transcription of surfactant $B$ and regulate various growth factors and growth factor receptors associated with alveolar septation and epithelial repair processes [5]. Repair of damaged lung in mature rats with elastase-induced emphysema following treatment with a retinoid, ATRA, was documented for the first time in 1997 by MASSARO and MASSARO [6].

The feasibility of using retinoids for the treatment of emphysema has been investigated in a short-term, placebo-controlled study (FORTE), funded through the National Heart, Lung and Blood Institute (National Institutes of Health, Bethesda, MD, USA). The patients received ATRA and 13-cis retinoic acid, but neither treatment showed an effect. This may have been the result of the compounds inducing metabolism of themselves. The results were sufficiently interesting that they justified the study of similar compounds that did not have this property [7].

Palovarotene (RO3300074) is a retinoid that selectively binds to the RAR- $\gamma$ subtype. In a replication of the MASSARO and MASSARO [6] study, palovarotene treatment showed a 30-50\% increase in alveolar area and an increase in arterial oxygen partial pressure $\left(\mathrm{Pa}_{1} \mathrm{O}_{2}\right)$ compared with control treatment in mature rats. Whether retinoids can produce similar effects in human emphysema needs to be studied [8]. Potential clinical outcomes of alveolar repair in this population with emphysema include slowing of the progression of the disease and partial restoration of lung function with improvement in gas transfer. Improvement in lung physiology may be accompanied by a reduction of symptoms of dyspnoea and an improvement of functional capacity. Such an outcome would suggest potential for modification of this disease.

\section{METHODS}

\section{Enrolment of participants}

Subjects with symptomatic emphysema secondary to AATD were recruited from 10 AAT registries in the Netherlands, UK, Sweden, Canada, Australia, New Zealand, Switzerland, Spain, Belgium and Denmark [9]. We included only males of more than 30 years of age and females of nonchildbearing potential with the Pi ZZ, Pi ZNull or Pi Null genotype. Other inclusion criteria included: ex-smokers or never-smokers with clinical diagnosis of emphysema confirmed by radiographic imaging (ex-smokers had to have stopped smoking for $\geqslant 6$ months prior to enrolment); subjects with transfer factor of the lung for carbon monoxide $(\mathrm{T} \mathrm{L}, \mathrm{CO})$ (or transfer coefficient of the lung for carbon monoxide, $\mathrm{KCO}$ ) $<70 \%$ of the predicted normal value and baseline post-brochodilator forced expiratory volume in $1 \mathrm{~s}$ (FEV1) $\leqslant 80 \%$ of the predicted normal value for sex, age, and height; subjects had to be off oral steroids $>28$ days prior to enrolment (e.g. prednisone, prednisolone). Subjects had to be never treated with AAT augmentation therapy.

Main exclusion criteria included: more than three exacerbations of pulmonary symptoms, requiring treatment with oral steroids or antibiotics, within 12 months prior to the study; observation of a solitary nodule in the lung requiring further medical intervention; subjects with giant bullous disease of $\geqslant 1 / 3$ of a hemithorax; psychiatric disorders requiring medication or hospitalisation, or with suicide attempt within the last 7 years; other significant medical conditions interfering with the subject's ability to perform the study tests; subjects using vitamin A or beta carotene, multivitamins containing vitamin A or beta carotene, or herbal preparations; hypertriglyceridaemia $\geqslant 300 \mathrm{mg} \cdot \mathrm{dL}^{-1}\left(3.4 \mathrm{~mol} \cdot \mathrm{dL}^{-1}\right)$ with therapy; unexplained weight loss of $\geqslant 10 \%$ of total body weight over the last 6 months or body mass index $<19 \mathrm{~kg} \cdot \mathrm{m}^{-2}$; history of allergy or sensitivity to retinoids; concurrent enrolment or participation in any other therapeutic clinical trial within the previous 30 days; subjects on lung transplant waiting list or who had undergone lung volume reduction surgery; and finally oral retinoid exposure in the past 12 months.

The study was approved by relevant local ethics review committees and was conducted in accordance with the Declaration of Helsinki and Good Clinical Practice guidelines, and all patients gave written informed consent.

\section{Study design}

This was an investigator-initiated, randomised, double-blind, placebo-controlled, parallel-group study conducted at 16 centres in 10 countries. Following a screening phase of up to 4 weeks, eligible patients were randomly assigned to receive two capsules per day of either palovarotene $2.5 \mathrm{mg}$ per capsule or placebo for 12 months, following the first meal of the day. At the end of the treatment period, subjects were followed up for an additional 4-week period for safety purposes.

After randomisation, patients were seen every 4 weeks at the clinics to receive study medication, a check for compliance, recording of the occurrence of any adverse events (AEs), to check for new concomitant treatments, for measurement of vital signs and for blood tests. The protocol specified a dose reduction in the event of mucocutaneous and other AEs higher than grade 2 . The study medication was put on hold for a maximum of 4 weeks or until symptoms had resolved or improved to grade 1 . Next, the study medication was resumed at 1 capsule $\cdot$ day $^{-1}(0.5 \mathrm{mg})$. If symptoms were not resolved or improved within 4 weeks the patient was withdrawn from the study.

\section{Study end-points}

Computed tomography (CT) scans of the lung were performed at baseline and at 6 and 12 months. A spiral CT scan was performed at each visit at a breath-hold at total lung capacity (TLC) [10]. Qualitative assessment of baseline CT scans was done by a radiologist at the site to confirm the presence of emphysema and to rule out the presence of lung nodules which may require medical intervention. Throughout the study, all CT images were analysed centrally (BioClinica, Leiden, the Netherlands) using PulmoCMS software (Medis Specials, Leiden, the Netherlands). 
The primary efficacy analysis was the comparison of volumeadjusted 15th percentile of the lung density histogram at 12 months adjusted for the baseline between the palovarotene and placebo [10]. This parameter was validated by classical studies performed by Gould et al. [11].

More detailed information on the lung function and CT assessments including standardisation of techniques across sites, has been reported previously [12].

Post-bronchodilator spirometry was performed according to the European Respiratory Society (ERS) spirometry guidelines [13]. A pre-bronchodilator test was also performed at screening only to establish the degree of reversibility.

Single breath TL,CO and KCO were performed following ERS guidelines, at least $30 \mathrm{~min}$ after bronchodilator administration [14].

TLC, functional residual capacity (FRC) and residual volume (RV) were measured by plethysmography. If a subject was unable to perform the required manoeuvres, or a site did not have access to a body plethysmograph, then the helium dilution method was used as an acceptable alternative. The same method had to be used with each subject and, where possible, performed by the same technician, throughout the study. Each test was performed at the same time of day $( \pm 2 h)$.

Spirometry, diffusing capacity and lung volume measurements were measured at baseline, 12, 24, 36, and 52 weeks.

During the study, the number of exacerbations of COPD was recorded as defined by an increase in respiratory symptoms for which the patient sought medical advice and for which a course of oral steroids and/or antibiotics was prescribed [15].

To guarantee the safety of all study subjects throughout the study, an independent Data Safety Monitoring Board reviewed semi-blinded safety summaries and all serious adverse events every 3 months. At the end of each review, a certificate of nonobjection to continue the study could be issued.

\section{Statistical analysis}

The primary efficacy variable was the change from baseline to 12 months of the 15th percentile of the lung density histogram adjusted for the lung volume, obtained by CT.

A pre-study feasibility check showed that a maximum of 260 AATD subjects (i.e. 130 subjects into each treatment group) should be enrolled into the study. This sample size provided a $70 \%$ power to detect a difference of $1 \mathrm{HU}$ in lung density (primary efficacy variable) at an $\alpha$-level of 0.05 using the twosided test with an assumed dropout rate of $15 \%$, for the active treatment group in comparison with placebo. The assumed common standard deviation of $3.0 \mathrm{HU}$ is based on the data from a previous study performed by DIRKSEN et al. [16].

To compare lung density values corrected for lung volume in the two treatment groups, a repeated-measures linear mixedeffects ANCOVA model was used. The model included fixed effects for treatment, visit, centre, and the subject as a random effect. The least squares mean value for the change from baseline in the 15th percentile of the lung density adjusted for the volume was calculated.
The intention-to-treat (ITT) population included all subjects randomised into the study who received at least 1 dose of the study medication. Subjects in the ITT population were analysed according to the treatment arm they were assigned to. Since treatment duration is a key factor for efficacy in this disease, subjects completing one year of treatment were also analysed separately.

In the placebo-treated study population, Spearman's correlation was calculated for the change in the 15th percentile point and the change in FEV1 or change in gas transfer.

Since REPAIR (Retinoid treatment of Emphysema in Patients on the $\alpha_{1}$-antitrypsin International Registry) was the first proof-of-mechanism study, subgroup analyses were undertaken in attempt to identify potential responders to treatment. In particular, baseline emphysema severity was considered to be an important factor in the evaluation of efficacy with palovarotene. In addition, we calculated the effect of a COPD exacerbation on the change from baseline in lung density by Chi-squared tests.

\section{RESULTS}

\section{Patients' disposition and baseline characteristics}

The study profile is shown in fig. 1. We screened 315 subjects on the AAT registries and 53 (17\%) failed screening and were not randomised into the study. Of the 262 eligible subjects, 129 were randomised to palovarotene and 133 to placebo. The number of subjects in the ITT population who completed the 12 month study was 110 in the palovarotene group and 117 in the placebo group; $15 \%$ and $12 \%$ of subjects prematurely discontinued from the study in the palovarotene and placebo group, respectively. There was a higher rate of permanent dose reduction to $2.5 \mathrm{mg} \cdot \mathrm{day}^{-1}$ in the palovarotene group $(11 \%)$ mainly because of expected mucocutaneous events versus $3 \%$ in the placebo group. The majority of first reported mucocutaneous events and pancreatic enzyme events occurred in the first quarter period of the study. Detailed information is provided in the online supplementary material.

Overall, demographics and disease severity for subjects at baseline were well matched between the groups (table 1). There was a small difference between the treatment groups in the number of never-smokers, with $21 \%$ in the palovarotene treatment group versus $11 \%$ in the placebo group, but this was not statistically significant. Overall the study included mainly patients with moderate-to-severe emphysema secondary to AATD. The most frequent major protocol deviations were: screening $T \mathrm{~L}, \mathrm{CO} \geqslant 70 \%$ pred $(9.8 \%$ in placebo and $10.1 \%$ in palovarotene) and screening FEV1 $>80 \%$ pred $(2.3 \%$ in placebo and $3.9 \%$ in palovarotene), but in all cases emphysema was present on CT images of the lung. Three additional subjects in the palovarotene group $(2.3 \%)$ had more than three exacerbations of their respiratory illness in the year before enrolment.

\section{Change from baseline in volume-adjusted 15th percentile lung density}

To compare the 15th percentile of the volume-adjusted lung density over time between the two treatment groups, the least square means were compared using a repeated-measure linear mixed-effect ANCOVA model. No additional covariates were identified in the linear mixed-effects model, thus the model 


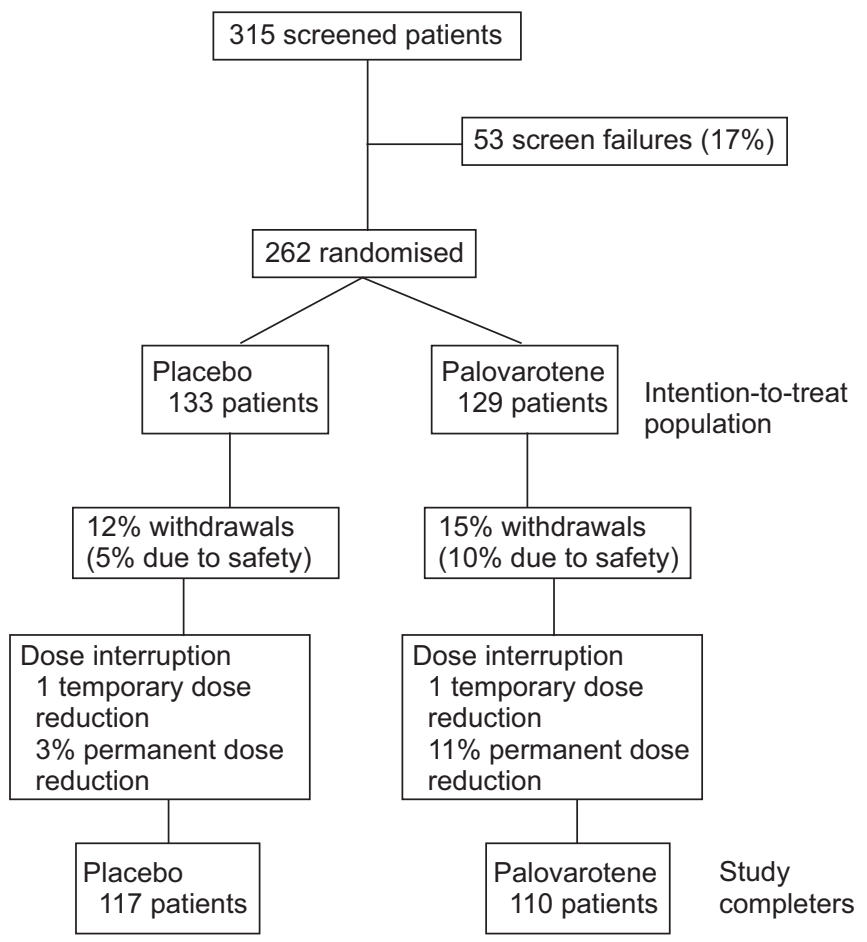

FIGURE 1. Patient disposition and study profile.

included only baseline density value, treatment, visit and treatment by visit interaction as fixed effects.

In the ITT population, the mean $\pm 95 \% \mathrm{CI}$ placebo-corrected difference was $-0.62 \pm 1.2 \mathrm{HU}$ by week $28(\mathrm{p}=0.36)$ and $-0.33 \pm$ 1.1 HU by week 52 from baseline, which was not statistically significant $(\mathrm{p}=0.88)$. In the completers population, the placebocorrected difference was $-0.45 \pm 0.9 \mathrm{HU}$ by week $28(\mathrm{p}=0.64)$ and $-0.25 \pm 0.7 \mathrm{HU}$ by week 52 from baseline $(p=0.94)$ (fig. 2 ).

We also calculated the change in relative area (RA) at -910 HU (RA -910) during the treatment period in the completers population. The mean $\pm 95 \%$ CI placebo-corrected difference was $2.3 \pm 6.1 \%$ by week 52 , which was not statistically significant $(p=0.78)$

\section{Change from baseline in FEV1 and lung volumes}

At week 52, the mean \pm SE decline from baseline in the completers population in postbronchodilator FEV1 was $40 \pm$ $14 \mathrm{~mL}$ in the palovarotene group and $50 \pm 13 \mathrm{~mL}$ in the placebo group $(p=0.86)$ (fig. 3$)$. Median FEV1 decline from baseline was $40 \mathrm{~mL}$ in the palovarotene group and $70 \mathrm{~mL}$ in the placebo group. In the placebo group, RV increased by $160 \pm 52 \mathrm{~mL}$ by week 52 compared to $100 \pm 49 \mathrm{~mL}$ in the palovarotene group (fig. 4). TLC in the completers population increased by $40 \pm 30 \mathrm{~mL}$ in the placebo group compared to a decrease of $-20 \pm 35 \mathrm{~mL}$ in the palovarotene group $(\mathrm{p}=0.07)$ with the curves appearing to separate after 6 months of treatment.

\section{Change from baseline in gas transfer}

Mean and median changes from baseline by week 52 in the completers population in haemoglobulin $(\mathrm{Hb})$-corrected $\mathrm{TL}, \mathrm{CO}$ were -0.22 and $-0.23 \mathrm{mmol} \cdot \mathrm{min}^{-1} \cdot \mathrm{kPa}^{-1}$ respectively, in the palovarotene group. In the placebo group, $1 \mathrm{yr}$ mean and

\begin{tabular}{lcc}
\hline TABLE 1 & Baseline subject characteristics & \\
& Placebo & Palovarotene \\
\hline & & \\
Subjects $\mathbf{n}$ & 133 & 129 \\
Age yrs & $53.9 \pm 8.6$ & $54.7 \pm 8.6$ \\
Sex male/female & $73 / 27$ & $71 / 29$ \\
Smoking status never/ex & $11 / 89$ & $21 / 79$ \\
Smoking exposure pack-yrs & $19.0 \pm 12.3$ & $19.2 \pm 13.1$ \\
Caucasian race & 100 & 100 \\
Body weight kg & $77.0 \pm 13.2$ & $77.4 \pm 12.8$ \\
FEV $\mathbf{1}$ & $1.56 \pm 0.6$ & $1.51 \pm 0.6$ \\
FEV $\mathbf{1} \%$ pred & $46.8 \pm 16.7$ & $46.4 \pm 16.8$ \\
FEV $\mathbf{1}$ FVC & $0.38 \pm 0.1$ & $0.38 \pm 0.1$ \\
TL,CO \% pred & $48.2 \pm 14.5$ & $48.2 \pm 15.1$ \\
KCO \% pred & $41.7 \pm 10.8$ & $42.2 \pm 11.9$ \\
SGRQ total score & $44.0 \pm 16.1$ & $46.5 \pm 15.8$ \\
15th percentile HU & $-962.0 \pm 14.0$ & $-959.3 \pm 15.3$ \\
TLC \% pred & $126.1 \pm 13.9$ & $122.3 \pm 13.9$ \\
RV \% pred & $187.6 \pm 52.5$ & $176.8 \pm 52.2$ \\
Dyspnoea index total score & $6.3 \pm 2.3$ & $6.2 \pm 2.6$ \\
ISWT m & $412.2 \pm 206.7$ & $409.6 \pm 194.7$ \\
Exacerbations $\mathbf{n}$ in previous year & $1.0 \pm 1.1$ & $1.1 \pm 1.1$ \\
Concomitant LABA+ICS+LAMA & 58 & 64 \\
\hline & & \\
\hline
\end{tabular}

Data are presented as mean $\pm \mathrm{SD}$ or $\%$, unless otherwise stated. FEV1: forced expiratory volume in $1 \mathrm{~s}$; \% pred: \% predicted; FVC: forced vital capacity; $T L, C O$ : transfer factor of the lung for carbon monoxide; $K \mathrm{co}$ : transfer coefficient of the lung for carbon monoxide; SGRQ: St George's Respiratory Questionnaire; TLC: total lung capacity; RV: residual volume; ISWT: incremental shuttle walking test; LABA: long-acting $\beta$-agonist; ICS: inhaled corticosteroids; LAMA: long-acting muscarinic agonist.

median changes from baseline in $\mathrm{Hb}$-corrected TL,CO were -0.23 and $-0.28 \mathrm{mmol} \cdot \mathrm{min}^{-1} \cdot \mathrm{kPa}^{-1}$, respectively (fig. 5).

Mean \pm SD change of the $\mathrm{Pa}, \mathrm{O}_{2}$ from baseline by week 52 in the completers population was $0.5 \pm 1.2 \mathrm{kPa}(\mathrm{p}=0.76)$ and the mean \pm SD change of the calculated alveolar-arterial oxygen delivery difference $\left(D A--a, \mathrm{O}_{2}\right)$ was $5 \pm 7 \%(\mathrm{p}=0.54)$.

\section{Exacerbations}

During the study, 91 patients in the placebo-treated group, and 83 patients in the treatment group experienced one or more exacerbations $(\mathrm{p}=0.515)$. In placebo-treated patients, the change in volume-adjusted 15 th percentile lung density correlated significantly with the frequency of exacerbations $(\mathrm{r}=0.22, \mathrm{p}=$ $0.017, \mathrm{n}=117)$, but this was not true of the palovarotene group $(\mathrm{r}=-0.171, \mathrm{p}=0.065, \mathrm{n}=117)$. The exacerbation frequency did not correlate with the change in FEV1 or gas transfer from baseline.

\section{DISCUSSION}

The REPAIR study represents the first investigator-initiated phase-II/III study of palovarotene, a drug designed to treat emphysema in patients with COPD. Patients with AATD were chosen for this first proof-of-concept study in males because of their young age and general lack of comorbidity, compared to usual COPD patients. While emphysema is the dominant 

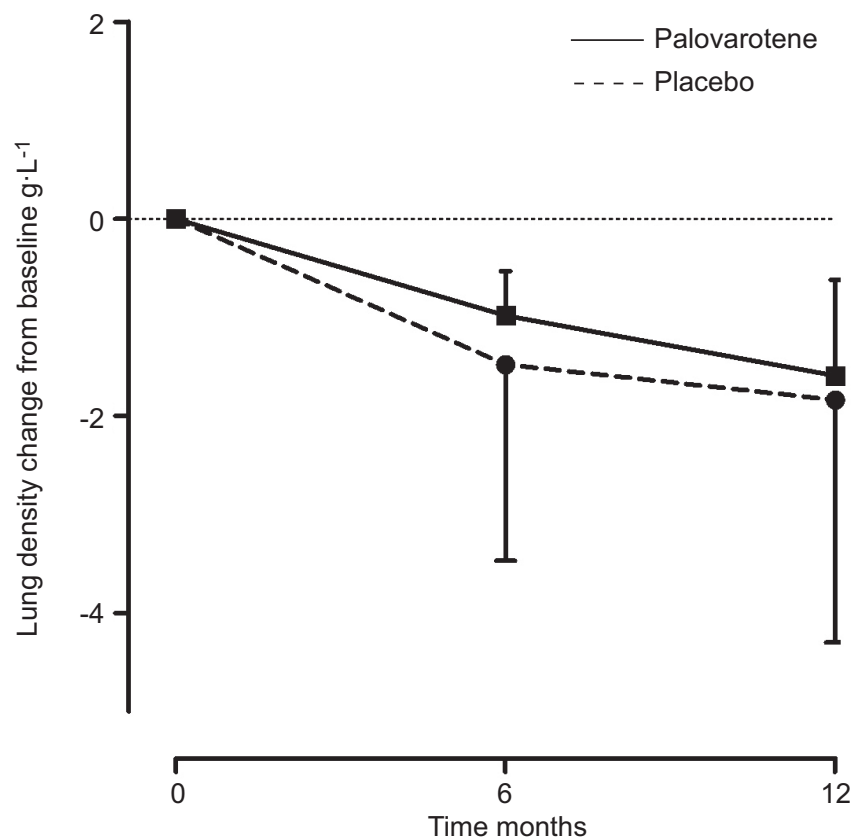

FIGURE 2. Primary efficacy parameter (study completers): least squares mean change from baseline in 15th percentile. Data are presented as mean $\pm 95 \% \mathrm{Cl}$.

pathological process, this population is also known for its relatively rapid progression compared to emphysema in general COPD [17]. The study mainly recruited patients with moderate-to-severe emphysema secondary to AATD and the baseline demographic and disease characteristics were comparable between the two treatment arms. In both treatment groups, mean volume-adjusted 15th percentile of lung density had declined from baseline by week 52 (-1.63 HU for palovarotene and $-1.81 \mathrm{HU}$ for placebo). Similar declines were seen in two other trials in the placebo-treated population [4, 16]. Our primary efficacy analysis showed that the least squares mean treatment difference in the volume-adjusted 15th percentile of lung density at week 52 was not significantly different between

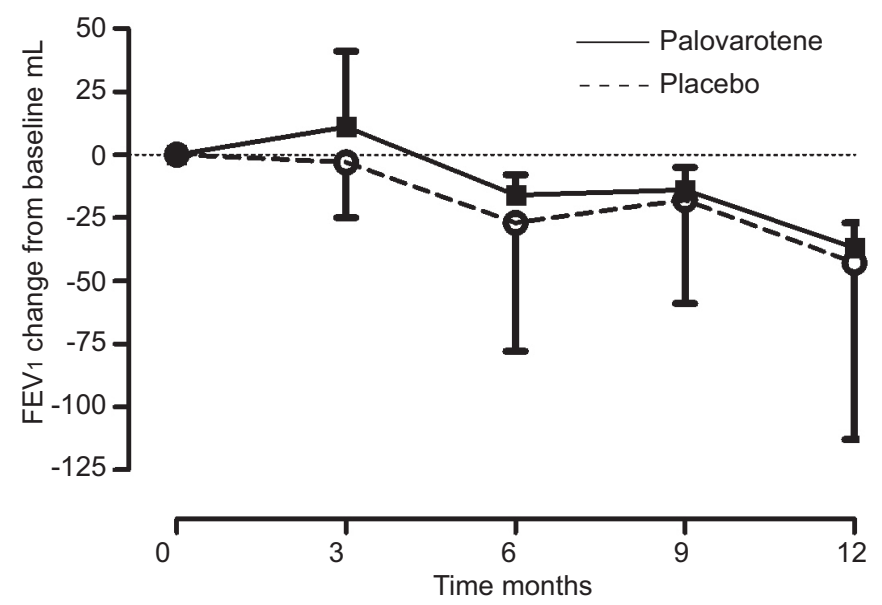

FIGURE 3. Least squares mean change from baseline in forced expiratory volume in $1 \mathrm{~S}\left(F E V_{1}\right)$ in the completers population. Data are presented as mean $\pm \mathrm{SE}$.

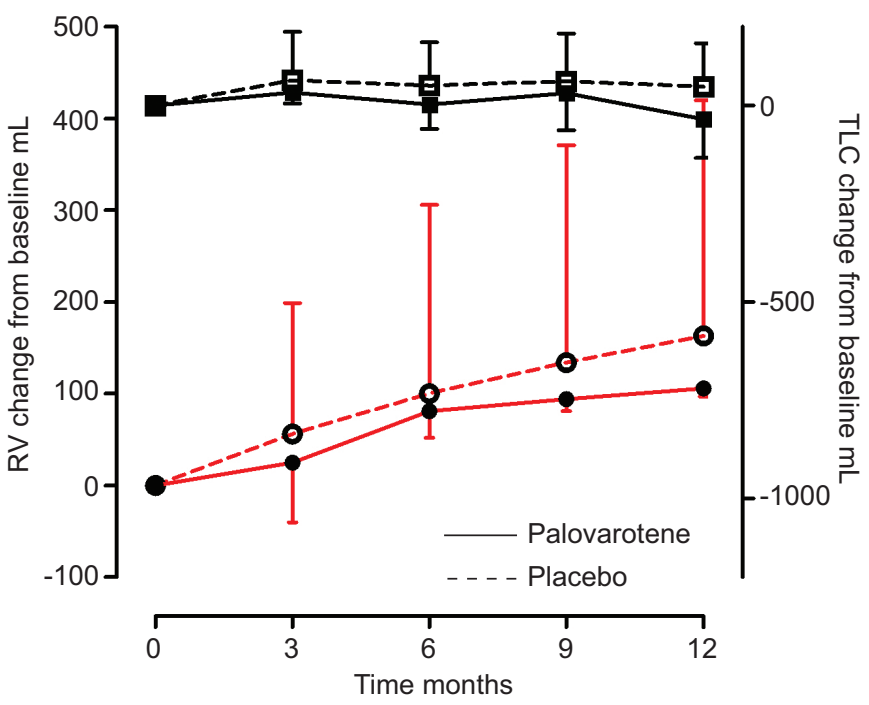

FIGURE 4. Least squares mean change from baseline in residual volume (RV) (left Y-axis; lower lines) and total lung capacity (TLC) (right Y-axis; upper lines) measured by plethysmography in the completers population. Data are presented as mean $\pm \mathrm{SE}$.

the treatment and placebo groups. The results based on the completer population analysis were similar to those for the ITT population analysis. Although, the study was insufficiently powered to detect statistically significant differences in secondary efficacy parameters (e.g. spirometry, diffusing capacity, lung volumes, and data not shown of exercise tests, dyspnoea index, and quality-of-life questionnaire score), we noted possible treatment-associated effects over time with palovarotene on several secondary efficacy parameters which did not reach statistical significance.

Recently, TANABE et al. [18] showed that exacerbations in patients with COPD $(n=26)$ accelerated the progression of emphysema measured by lung densitometry compared to stable COPD patients $(n=34)$. They explained their result by a computed

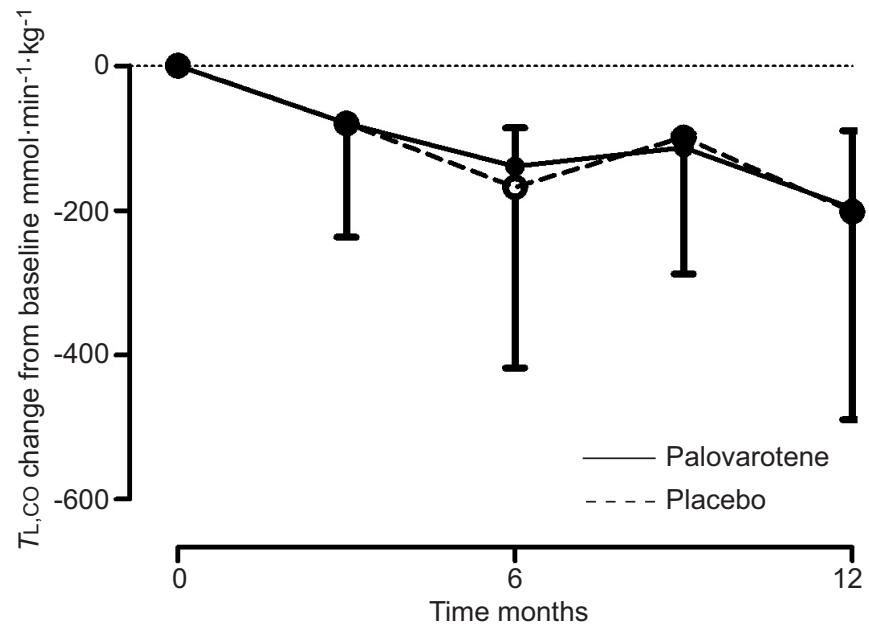

FIGURE 5. Least squares mean change from baseline in haemoglobulincorrected transfer factor of the lung for carbon monoxide $(T L, C O)$, in the completers population. Data are presented as mean $\pm \mathrm{SE}$. 
model in which it was shown that the number of alveolar walls was reduced in subjects with exacerbations. In our larger population, the decline in lung density in the placebo-treated population $(n=117)$ correlated significantly with exacerbation rates during the study period. However, as in the report by TANABE et al. [18], we could not find a correlation between exacerbation rate and a change in any of the lung function parameters.

Additional investigations are needed to explore whether trends in improved lung function between the two treatment groups represented a true finding of clinical importance. Based on available sub-chronic and chronic toxicology studies in rats and dogs of palovarotene at study start, the daily $5 \mathrm{mg}$ dose was the highest dose that could be used for long-term treatment in patients. This dosage was also based on the effective range of exposures seen in a rat elastase animal model, as well as on the tolerability of the compound in clinical pharmacology studies of healthy human volunteers. The pharmacokinetic data of palovarotene obtained in the REPAIR study showed the expected area-under-the-curve exposure results (data not shown). It is likely that longer treatment with palovarotene is required to show a greater treatment effect on our primary outcome measure since the progression of emphysema in ex-smokers may be slow and eventually may also show a treatment effect on lung function parameters.

Patients with moderate emphysema might be expected to respond better to palovarotene than those with severe emphysema. This is based on the consideration that 1) emphysema progression would be slower and 2) less-damaged areas of emphysema may repair more quickly. We have chosen gas transfer at baseline rather than lung density (to prevent regression to the mean) to identify patients with mild-to-moderate emphysema. We carried out a sub-analysis for patients with KCO above and below the median value. However, this exercise also failed to detect a treatment effect.

AATD-related emphysema is almost always present in the lower lung lobes [17]. These are the areas with the best blood perfusion and therefore best drug supply to lung parenchyma. They may also be the lobes with the most active inflammation and alveolar destruction compared with upper lung lobes. We performed more detailed exploratory analyses of efficacy in subgroups with changes in upper, middle and lower lung lobe identified by calculating the slope of the regression line of the lung density values over 12 lung partitions [19]. After 1 yr density declined by $-1.2 \mathrm{HU},-1.8 \mathrm{HU}$ and $-1.4 \mathrm{HU}$ from baseline in the placebo group in the apical, middle and basal regions, respectively. Progression in the middle part of the lung was largest as previously reported by PARR et al. [18] in a different study population, but palovarotene did not significantly reduce progression in the middle region, nor in the other regions.

In general, the safety profile of AATD emphysema subjects receiving $5 \mathrm{mg}$ of palovarotene daily for 12 months was consistent with the known effects of palovarotene and retinoids, and the drug was well tolerated in this group of subjects.

We conclude that this study failed to show a significant difference in lung volume-adjusted 15th percentile point density. Interestingly, palovarotene seemed to protect against the effect of exacerbations of COPD on lung density 15th percentile point, a measure of progression of emphysema. $1 \mathrm{yr}$ of treatment may be insufficient to obtain clinically relevant benefits with palovarotene. Concordant effects were seen over time with palovarotene on most of the efficacy parameters. Although statistical differences with placebo were not found, these results might suggest a pharmacological effect of palovarotene. However, a clear phenotype of responders to palovarotene remains to be identified.

\section{STATEMENT OF INTEREST}

Statements of interest for R.T. Abboud, B.G. Cooper, M. Decramer, W.A. MacNee, A. Rames, R.A. Stockley, B.C. Stoel and J. Stolk can be found at www.erj.ersjournals.com/site/misc/statements.xhtml

\section{ACKNOWLEDGEMENTS}

Author affiliations are as follows. J. Stolk: Dept of Pulmonology, Leiden University Medical Center, Leiden, The Netherlands; R.A. Stockley, B.G. Cooper: Lung Investigation Unit, Queen Elizabeth Hospital, Birmingham, UK; B.C. Stoel: Dept of Radiology, Leiden University Medical Center, Leiden, The Netherlands; E. Piitulainen: Dept of Respiratory Medicine, Malmö University Hospital, Malmö, Sweden; N. Seersholm: Dept of Pulmonary Medicine Y, Gentofte Hospital, Hellerup, Denmark; K.R. Chapman: Asthma and Airway Centre, Toronto Western Hospital, Toronto, Canada; J.G.W. Burdon: Dept of Respiratory Medicine, St Vincent's Hospital, Fitzroy, Australia; M. Decramer: Dept of Pneumology, Gasthuisberg University Hospital, Leuven, Belgium; R. T. Abboud: Respiratory Medicine Division, University of British Columbia, Vancouver General Hospital, Vancouver, BC, Canada; G.P.M. Mannes: Dept of Pulmonology, Twenteborgziekenhuis, Almelo, The Netherlands; E.F. Wouters: Dept of Respiratory Medicine, University Hospital Maastricht, Maastricht, The Netherlands; J.E. Garrett: Dept of Medicine, Middlemore Hospital, Auckland, New Zealand; J.C. Barros-Tizon: Servicio de Neumologia, C. Hospital Xeral-Cies, Vigo, Spain; E.W. Russi: Pulmonary Division, University Hospital, Zurich, Switzerland; D.A. Lomas: Dept of Medicine, University of Cambridge, Cambridge Institute for Medical Research, Cambridge, UK; W.A. MacNee: ELEGI/Colt Research Laboratories, MRC/UOE Centre for Inflammation Research, Edinburgh, UK; A. Rames: Hoffmann-La Roche, Basel, Switzerland.

We want to thank all study teams from the study sites for their excellent work and dedication during the conduct of this study.

\section{REFERENCES}

1 McDonough JE, Yuan R, Suzuki M, et al. Small-airway obstruction and emphysema in chronic obstructive pulmonary disease. N Engl J Med 2011; 365: 1567-1575.

2 Damiano VV, Tsang A, Kucich U, et al. Immunolocalization of elastase in human emphysematous lungs. J Clin Invest 1986; 78 : 482-493.

3 Global Initiative for Chronic Obstructive Lung Disease. Global Strategy for diagnosis and prevention of chronic obstructive pulmonary disease. NHLBI/WHO workshop report. Bethesda, National Heart, Lung and Blood Institute, 2001; NIH Publication No. 2701.

4 Stockley RA, Parr DG, Piitulainen E, et al. Therapeutic efficacy of $\alpha_{1}$-antitrypsin augmentation therapy on the loss of lung tissue: an integrated analysis of 2 randomised clinical trials using computed tomography densitometry. Respir Res 2010; 11: 136.

5 Belloni PN, Garvin L, Mao CP, et al. Effects of all-trans-retinoic acid in promoting alveolar repair. Chest 2000; 117: 235S-241S.

6 Massaro GD, Massaro D. Retinoid acid treatment abrogates elastase-induced pulmonary emphysema in rats. Nat Med 1997; 3: 675-677. 
7 Roth MD, Connett JE, D'Armiento JM, et al. Feasibility of retinoids for the treatment of emphysema study. Chest 2006; 130: 1334-1345.

8 Massaro D, Massaro GD. Lung development, lung function, and retinoids. N Engl J Med 2010; 362: 1829-1831.

9 Stockley RA, Luisetti M, Miravitlles M, et al. Ongoing research in Europe: Alpha One International Registry (AIR) objectives and development. Eur Respir J 2007; 29: 582-586.

10 Stoel BC, Putter H, Bakker ME, et al. Volume correction in computed tomography densitometry for follow-up studies on pulmonary emphysema. Proc Am Thorac Soc 2008; 5: 919-924.

11 Gould GA, MacNee W, McLean A, et al. CT measurements of lung density in life can quantitate distal airspace enlargement - an essential defining feature of human emphysema. Am Rev Respir Dis 1988; 37: 380-392.

12 Stolk J, Cooper BG, Stoel B, et al. Retinoid treatment of Emphysema in Patients on the Alpha-1 International Registry. The REPAIR study: study design, methodology and quality control of study assessments. Ther Adv Respir Dis 2010; 4: 319-332.

13 European Respiratory Society. Standardized lung function testing. Lung volumes and forced ventilatory flows. 1993 update. Eur Respir J 1993; 6: 5-40.
14 Cotes JE, Chinn DJ, Quanjer PH, et al. Standardization of the measurement of transfer factor (diffusing capacity). Report Working Party Standardization of Lung Function Tests, European Community for Steel and Coal. Official Statement of the European Respiratory Society. Eur Respir J 1993; 6: Suppl. 16, 41-52.

15 Rodríguez-Roisin R. COPD exacerbations. 5: Management. Thorax 2006; 61: 535-544.

16 Dirksen A, Dijkman JH, Madsen F, et al. A randomized clinical trial of $\alpha_{1}$-antitrypsin augmentation therapy. Am J Respir Crit Care Med 1999; 160: 1468-1472.

17 Parr DG, Sevenoaks M, Deng C, et al. Detection of emphysema progression in $\alpha 1$-antitrypsin deficiency using CT densitometry; methodological advances. Respir Res 2008; 9: 21.

18 Tanabe N, Muro S, Hirai T, et al. Impact of exacerbations on emphysema progression in chronic obstructive pulmonary disease. Am J Respir Crit Care Med 2011; 183: 1653-9.

19 Bakker ME, Putter H, Stolk J, et al. Assessment of regional progression of pulmonary emphysema with CT densitometry. Chest 2008; 134: 931-937. 